\title{
LOS CAMBIOS AMBIENTALES DEL HOLOCENO MEDIO/ HOLOCENO TARDÍO EN EL DESIERTO DE SONORA Y SUS IMPLICACIONES EN LA DIVERSIFICACIÓN DEL YUTO- AZTECANO Y LA DIFUSIÓN DEL MAÍZ
}

\author{
ENVIRONMENTAL CHANGE IN THE MIDDLE HOLOCENE/LATE HOLOCENE \\ IN THE SONORAN DESERT AND ITS IMPLICATIONS REGARDING THE \\ DIVERSIFICATION OF UTO-AZTECAN AND THE DIFFUSION OF MAIZE
}

\author{
John Carpenter Slavens ${ }^{*}$ y Guadalupe Sánchez ${ }^{* *}$
}

\begin{abstract}
En este trabajo presentamos un modelo para explicar la bifurcación inicial de la familia lingüística yutoazteca, la diversificación subsecuente de la rama sonorense/sureño y la difusión inicial del maíz al noroeste de México y el suroeste de Estados Unidos. El Holoceno medio (también conocido como el altitermal) está fechado aproximadamente entre los años de 7500 a 4500 antes del presente (5500-2500 a.C.). Este período se caracteriza por condiciones climáticas adversas durante el cual el desierto de Sonora quedó casi abandonado. Sugerimos que los grupos yutoaztecanos fueron los primeros norteños en obtener el maíz (a través del occidente). También proponemos que estos grupos hablantes del protoyutoaztecasonorense salieron de su refugio altitermal, ubicado en la zona serrana del noreste de sinaloa, sureste de sonora y sureste de chihuahua y recolonizaron el desierto de sonora.

Palabras claves: difusión del maíz, yutoaztecanos, altitermal.
\end{abstract}

In this paper we present a model to explain the initial bifurcation of the linguistic uto-aztecan family, its subsequent diversification of the sonoran/southern branch and the initial diffusion of maize to northwest mexico and the southwest of the u.s. the middle holocene (also known as the altithermal) is aproximately dated to the years between 7500 and 4500 before the present (55002500 bce). This period is characterized by adverse climactic conditions during which time the sonoran desert remained almost abandoned. We sugest that these uto-aztecan groups were the first northerners to obtain maize (via west mexico). Additionally, we propose that these groups of proto-sonoran-uto-aztecan speakers left their altithermal refuge, located in the foothill/mountain zone of northeastern sinaloa, southeastern sonora and southwestern chihuahua and recolonized the sonoran desert.

Key words: diffusion of maize, uto-aztecan, altithermal.

\section{Introducción}

El estado de Sonora refleja una enorme historia de ocupación humana representada por varios desarrollos sociales que comenzaron desde finales del Pleistoceno y continuaron casi sin interrupción hasta tiempos recientes. Los grupos que habitaron el desierto de Sonora no solo sobrevivieron, sino florecieron durante aproximadamente cien siglos adaptados a un ambiente severo, pero que contiene un enorme repertorio de plantas y animales importantes para la supervivencia humana. Los grupos humanos que habitaron Sonora tuvieron que enfrentar por lo menos dos cambios climáticos drásticos y de larga duración. El primero durante el Pleistoceno terminal conocido como Youngest Dryst, y posteriormente durante el Holoceno medio conocido como Altitermal. Durante estos períodos de extrema desertificación ocurrieron abandonos esporádicos del desierto, aunque las poblaciones vuelven a ocupar el desierto y logran adaptarse y permanecer en las zonas desérticas e inclusive desarrollar comunidades sedentarias de agricultores incipientes. Este trabajo pretende investigar a través de las modificaciones de algunos paisajes sonorenses y los cambios climáticos que definen el Holoceno tardío, el papel que tuvo Sonora y los grupos hablantes de yutoaztecas en la propagación del cultivo de maíz al suroeste de Estados Unidos que ocurrió hace por lo menos 4000 años.

\footnotetext{
* Instituto Nacional de Antropología e Historia, Centro INAH, Sonora, México. Correo electrónico: chichimecatl@ hotmail.com

** Universidad Nacional Autónoma de México (UNAM), Instituto de Geología, Estación Región del Noroeste (ERNO), México. Correo electrónico: guadalupe_sanchez_miranda@hotmail.com
} 


\section{El paisaje sonorense}

El desierto de Sonora abarca alrededor de 360.000 kilómetros cuadrados en ambos lados de la frontera internacional, incluyendo la gran mayoría de Sonora, la península de Baja California, el suroeste del estado de Arizona y el extremo sureste de California en los EE.UU. Como desierto, se caracteriza por su extrema aridez, con un promedio de precipitación anual que varía entre $50 \mathrm{~mm}$ y $300 \mathrm{~mm}$, dependiendo de la zona. La precipitación refleja una distribución bimodal, con un poco más de lluvia asociada a los chubascos del verano que a las equipatas invernales. Las temperaturas son extremas, variando entre -5 centígrados en el invierno, y alcanzan 45 o más grados entre junio y septiembre. Es un desierto tropical único en el mundo, con una biodiversidad vegetal y animal relativamente alta (Nabhan, 1985, 1989; Turner y Brown, 1994:182)

Los cambios climáticos y de vegetación en Sonora han sido unidireccionales, con una tendencia a la desertificación que comenzó durante el Pleistoceno terminal hasta conformar el actual desierto de Sonora (Nabhan, 1985; Vandenver, 1990). Los restos botánicos recuperados de los nidos de neotomas representan uno de los mejores registros para estudiar el clima y la sucesión vegetal en la zona (Van Devender, 1990). Estos estudios junto con los pocos registros de polen que existen para Sonora y áreas vecinas (Davis y Anderson, 1987), y por lo menos un estudio de restos de carbón e isótopos de oxígeno en los huesos de los mamíferos del Pleistoceno de Sonora (Núñez et al., 2010) han servido como base para reconstruir preliminarmente la historia climática del desierto de Sonora durante los últimos 16000 años.

La evidencia ambiental que tenemos hasta la fecha señala que en la región noroeste de Sonora a finales del Pleistoceno existía un bosque de piñón y táscate con yuca y pastizales abiertos. Esta comunidad vegetativa comenzó a deteriorarse alrededor del 11000 antes del presente; los táscates (Juniperus californica) permanecieron en el paisaje hasta 8900 años antes del presente (Van Devender et al., 1987). En las partes más altas cerca de la Sierra Madre el pino se comporta de la misma forma que los táscates y comienza a desaparecer 8700 años antes del presente. Los registros de polen muestran un aumento de mesquite durante este horizonte y a partir del 8000 antes del presente el clima y la vegetación es muy similar a la actual. Bosques de leguminosas con lluvias monsónicas abundantes en el verano y veranos muy calientes comienzan a ser la regla del desierto de Sonora (Van Devender et al., 1990).

El Holoceno medio (también conocido como el Altitermal) está fechado aproximadamente entre los años de 7500 a 4500 antes del presente (55002500 a.C.). Iinicialmente fue definido por Antevs $(1948,1955)$ como un período de estrés climático de dimensiones macrorregionales representado por temperaturas muy altas y un descenso considerable de las lluvias. La severidad de las condiciones ambientales varió de región en región; en algunas partes los grupos humanos tuvieron que adaptarse a las sequías, otros lugares casi tuvieron que ser abandonados por completo y la gente tuvo que refugiarse en lugares a mayor elevación o con microclimas mucho más amigables (Betancourt 1990; Davis 1984; Holliday 1989; Martin 1963; Meltzer 1991; Van Devender 1990). En el desierto de Sonora el Altitermal se caracteriza por la falta de depósitos y registros arqueológicos debido a que la gran sequía erosionó la mayoría de los depósitos y existe un hiato estratigráfico que parece corroborar los modelos que plantean condiciones ambientales inhóspitas (Berry y Berry, 1986; Mabry, 1998c, 1998d).

\section{La familia lingüística yutoaztecana y el Holoceno medio (Altitermal)}

La familia lingüística yutoaztecana es una de las familias más importantes del continente de Norteamérica, con una distribución extendiéndose desde la Gran Cuenca en el norte hasta Nicaragua en el sur (Figura 1). Diversas áreas han sido identificadas como la ubicación de la cuna de los protoyutoaztecas (PUA). Se ha propuesto desde la meseta de Columbia en el extremo noroeste de EE.UU. (Hopkins, 1965), el norte de California (Nichols, 1981), la Gran Cuenca (Goss, 1977), y desde el norte de las Montañas Rocosas, hasta los altos de los altos del Mogollon (Arizona-Nuevo México) y la Sierra Madre Occidental (Fowler, 1983; Romney, 1957), los desiertos de la frontera entre Arizona-Sonora (Lamb, 1958), el sur de California (Miller, 1994), o tal vez en el altiplano de México central (Hill, 2001) como lugares posibles del primer grupo yutoaztecano.

Basándose en 27 términos cognados relacionados con la flora y fauna, los cuales fueron parte 


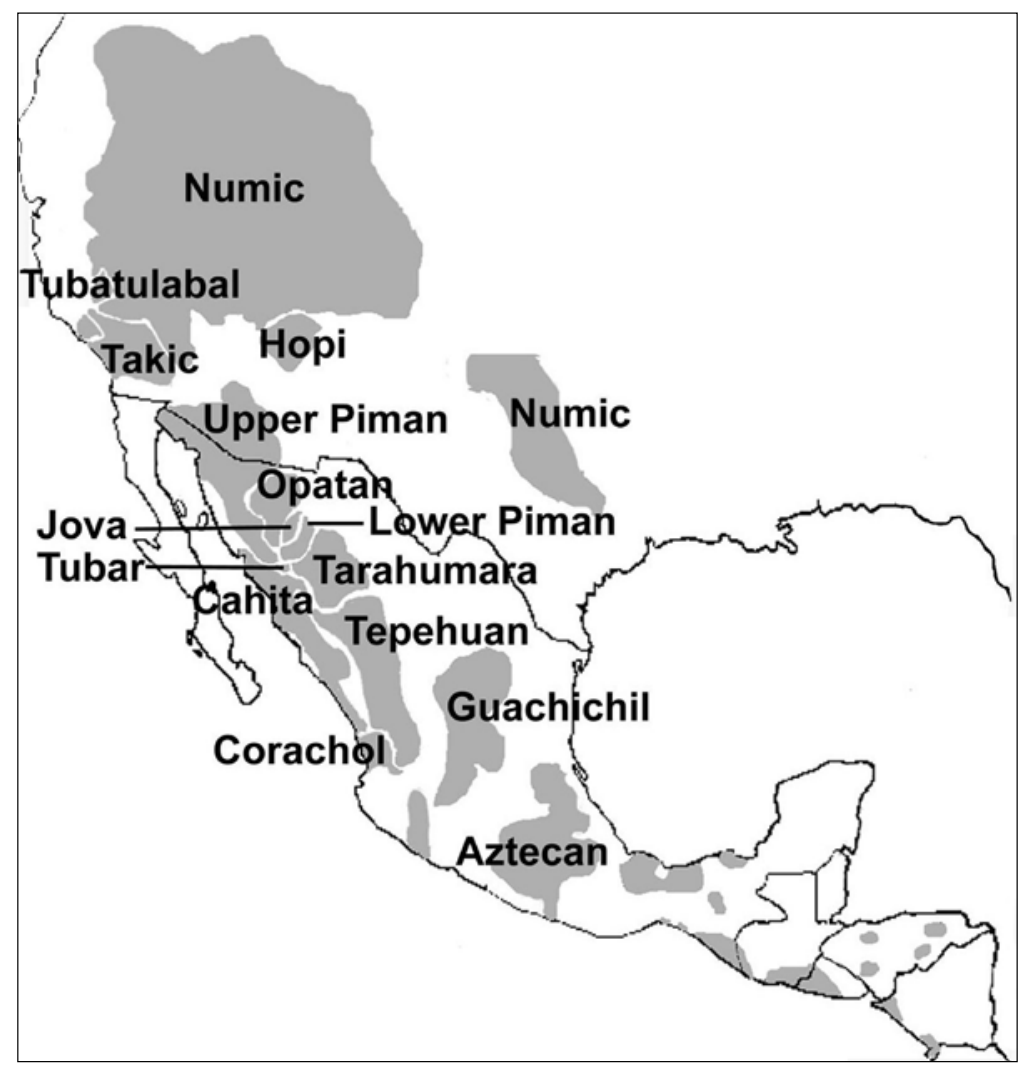

Figura 1. La familia yutoaztecana en Norteamérica.

integral del conocimiento biológico folklórico de los grupos hablantes de PUA en un momento antes de la diversificación entre las ramas norteñas y sonorenses, entre aproximadamente 4000 y 2000 a.C., Catherine Fowler (1983) los ubica en un lugar de elevación media con un ecotono de bosques y pastizales mixtos de la serranía norte de la Sierra Madre Occidental y la provincia de serranías y valles del este de Arizona y el oeste de Nuevo México.

Proponemos que la bifurcación de la familia yutoaztecana no solo coincide con el período Holoceno medio, sino que fue resultado de los cambios climáticos asociados a esta época. En nuestra perspectiva, ciertos grupos abandonaron la cuna original propuesta por Fowler: algunos migrando hacia la Gran Cuenca buscando refugio en mejores condiciones ambientales y formaron la rama norteña inicial; mientras que otros se movieron hacia el sur y al este donde los efectos del Altitermal no se sintieron con tanta rudeza y los humanos, plantas y animales pudieron vivir en la
Sierra Madre Occidental, constituyendo la rama que se reconoce como sonorense (Carpenter et al., 1996, $1998,2002,2005,2001)$. Es muy probable que la rama sonorense de la familia yutoaztecana sureña se desarrolló durante el período Arcaico Medio (ca. 5000 a 2000/1500 a.C.) entre poblaciones que ocuparon las serranías de la Sierra Madre Occidental del sur de Sonora y Chihuahua (Miller, 1995:5).

\section{Proposiciones sobre el origen del maíz}

Hoy se pueden considerar dos modelos existentes para explicar el origen del maíz. El modelo Tehuacán (o altiplano) considera que el maíz se originó en el altiplano por medio de la hibridación introgresiva entre Tripsacum, teosinte (Zea spp.), y una raza extinta de maíz (MacNeish y Eubanks, 2000; Manglesdorf y Reeves, 1939; Manglesdorf et al., 1981; Manglesdorf, 1983, 1986). Como alternativa, el modelo del río Balsas (o tierras bajas) propone que el maíz se derivó directamente 
de teosinte (Benz, 1999; Doebley, 1990; Doebley et al., 1984, 1987; Iltis, 1983, 1987). El teosinte se distribuye naturalmente del lado del océano Pacífico desde Nicaragua hasta unas cuencas aisladas en el estado de Chihuahua. Sin embargo, la mayor diversidad de taxa se localiza en las cuencas del río Balsas y río Lerma. Sobre la base de información bioquímica, el río Balsas ha sido identificado como el territorio original de la evolución del maíz (Benz, 1999; Doebley et al., 1984, 1987). El reconocimiento de la existencia de un teosinte perenne, Zea diploperennis, en Jalisco apoya el modelo Balsas, donde se propone que el origen del maíz ocurrió en la vertiente del Pacífico. Según la Teoría de Transmisión Sexual Catastrófica (Catastrophic Sexual Transmission Theory) (Iltis, 1983, 1987), esta mutación sexual transformó la inflorescencia masculina de teosinte a un elote femenino.

Después de su etapa inicial de evolución, el maíz se dispersó a varias regiones, diversificándose en dos ramas distintas, reflejando cada una un desarrollo in situ (Benz, 1999:32; Sánchez
González, 1994). Utilizando como base el índice de la frecuencia cromosómica del grano (chromosome knob frequency) se ha postulado que la ramificación relevante del maíz para el noroeste de México y el suroeste de EE.UU. debió haberse originado en la región de Colima y Jalisco, donde se desarrolló la raza de maíz Reventador; posteriormente se trasladó a la planicie costera de Nayarit, donde apareció la raza Jala, y de allí a la costa de Sinaloa y Sonora, donde se desarrolló el maíz Chapalote (Benz, 1994:32-33) (Figura 2).

Hasta la fecha la única manera como se puede elaborar una cronología tentativa para la domesticación de maíz es basándose en la presencia de teosinte y maíz temprano en contextos arqueológicos. Se ha documentado teosinte en depósitos fechados alrededor de 5000 a.C. en Zohapilco/Tlapacoya (Niederberger, 1976, 1979), en la cuenca de México, los restos de maíz más tempranos que han sido reportados recientemente por Piperno y Flannery (2001) y Benz (2001) tienen una fecha de 4200 a.C. procedentes de la cueva de Guilá Naquitz, en Oaxaca. Mientras que, en varias regiones de México,

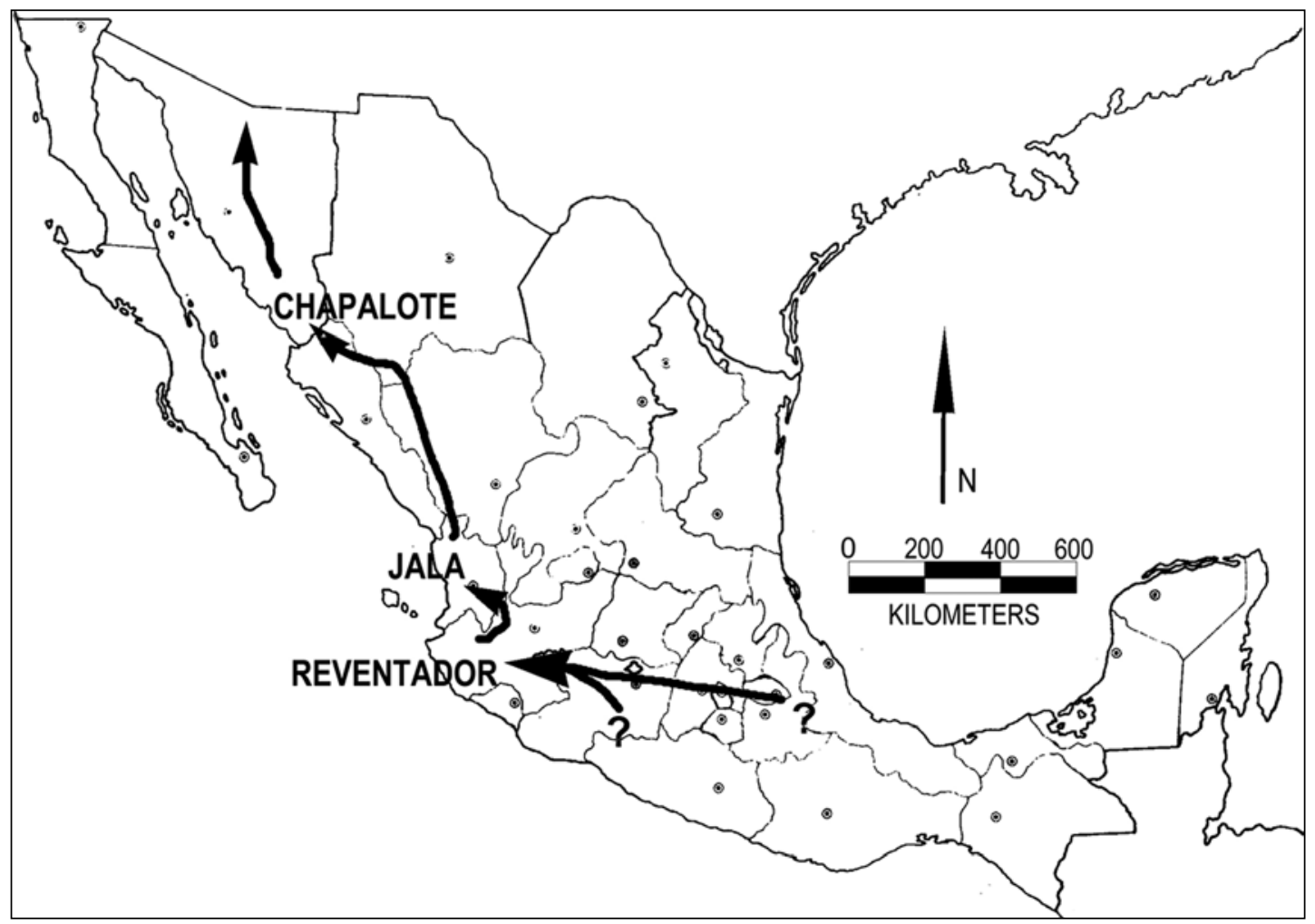

Figura 2. Diversificación del maíz hacia el Norte de México. 
desde Tamaulipas hasta Chiapas, las muestras de maíz fechadas por radiocarbono varían entre aproximadamente 2500 y 1500 a.C. (McClung de Tapia, 1997: 38).

\section{El cultígeno mesoamericano Zea mays spp. $y$ su desplazamiento al norte}

En la última década se ha reconocido la existencia del período de Agricultura Temprana que representa una etapa de agricultura precerámica en las zonas áridas del norte. Este período está representado por más de una docena de sitios en la cuenca de Tucson en Arizona, La Playa en Sonora, cerro Juanaqueña en Chihuahua y media docena de sitios en Nuevo México (Herr, 2009: 1) (Figura 3). Esta adaptación humana de aldeas agrícolas en las planicies aluviales de los ríos con tecnología de control de agua como canales, campos con bordos (Figura 4) y cercas vivas, solo pudo ser posible por la llegada del cultígeno maíz al norte de México. El maíz fue inventado por el hombre de su ancestro silvestre el teosinte de la zona del Balsas (Zea mays ssp. Parviglumins) algunos milenios antes de que llegara al norte (Benz, 1999; Doebley, 1990; Doebley et al., 1984, 1987; Iltis, 1983, 1987). El macrofósil de maíz más viejo de Mesoamérica que se conoce fue recuperado en la cueva Guilá Naquitz en Oaxaca y tiene una fecha calibrada de 4280 a.C. (Piperno y Flannery, 2001 y Benz, 2001). Con múltiples fechamientos directos firmemente se puede establecer que el maíz (Zea mays spp.) fue introducido al suroeste de Estados Unidos por lo menos en el año 2100 a.C. (Merrill et al., 2009; Mabry et al., 2008). Con las fechas que se tienen hasta el momento se puede asumir que la difusión del maíz hacia el norte tomó por lo menos 2200 años. Las evidencias del maíz temprano en el suroeste de Estados Unidos provienen de tres sitios abiertos y tres cuevas en Arizona y el oeste de Nuevo México, variando en elevación de 700-220 m, lo que sugiere que Zea mays tiene una gran plasticidad para adaptarse a diversos nichos ecológicos, esta capacidad de adaptación explica su rápida expansión. Existen varias teorías para explicar los mecanismos involucrados en la transmisión y adopción del maíz al norte de México; algunos estudios sugieren que fueron emigrantes agricultores de Mesoamérica, otros que la transmisión del maíz ocurrió poco a poco saltando de comunidad en comunidad.
Estas fechas sugieren que el maíz llegó al desierto de Sonora 1500 años después de su domesticación inicial, pero cabe señalar que las fechas con las que contamos para el maíz temprano en otras regiones de México hasta las cercanas al centro original, son equivalentes a las del desierto de Sonora, por lo que el maíz se dispersó rápidamente hacia el norte.

Los modelos interpretativos para la introducción de maíz en el noroeste de México y el suroeste de los EE.UU. pueden agruparse en dos categorías generales: 1) los modelos basados en que la adopción de maíz y otros cultígenos originados en el centro de México se debió a grupos de recolectores-cazadores locales; o 2) los modelos que argumentan la introducción de cultígenos vía grupos migratorios. Sin duda, los modelos basados en la difusión son los más populares, ya que sugieren que el maíz fue adoptado por grupos indígenas de recolectorescazadores como una estrategia para minimizar la incertidumbre ambiental (Hard, 1986; Wills, 1988, 1990) provocada por el aumento poblacional y/o disminución de los recursos naturales, o tal vez, el maíz fue incorporado por grupos ya semisedentarios que estaban ocupando áreas donde diversos ecotonos pudieron ser explotados fácilmente, como Paul y Suzy Fish (Fish et al., 1990) han sugerido para la cuenca de Tucson.

Sin embargo, algunos otros (Berry y Berry, 1986; Huckell, 1990, 1995) reconocen la existencia de una discontinuidad en la tradición Cochise, e identifican el inicio de la fase San Pedro alrededor de 1500/1200 a.C., con la llegada de grupos de agricultores de México. R.G. Matson (1991, 1999) sugiere una afinidad entre la fase San Pedro y Western Basketmaker II, y propone que la tradición Western Basketmaker II se derivó de la tradición San Pedro por medio de una migración poblacional hacia la meseta de Colorado después de que se desarrollara una variedad de maíz adaptada al frío.

Nosotros proponemos que la introducción de maíz en el noroeste de México y el suroeste de los EE.UU. puede ser atribuida a la dispersión de grupos hablantes de yutoazteca que ocurrió durante los primeros siglos inmediatamente después del período Altitermal (Holoceno Medio). Ernest Antevs (1948, 1955) definió el período Altitermal como el lapso de tiempo caracterizado por sus altas temperaturas y poca precipitación entre aproximadamente el año 5000 y 2500 a.C., aunque algunos investigadores siguen debatiendo la severidad de las condiciones ambientales; parece ser que por lo menos en el 


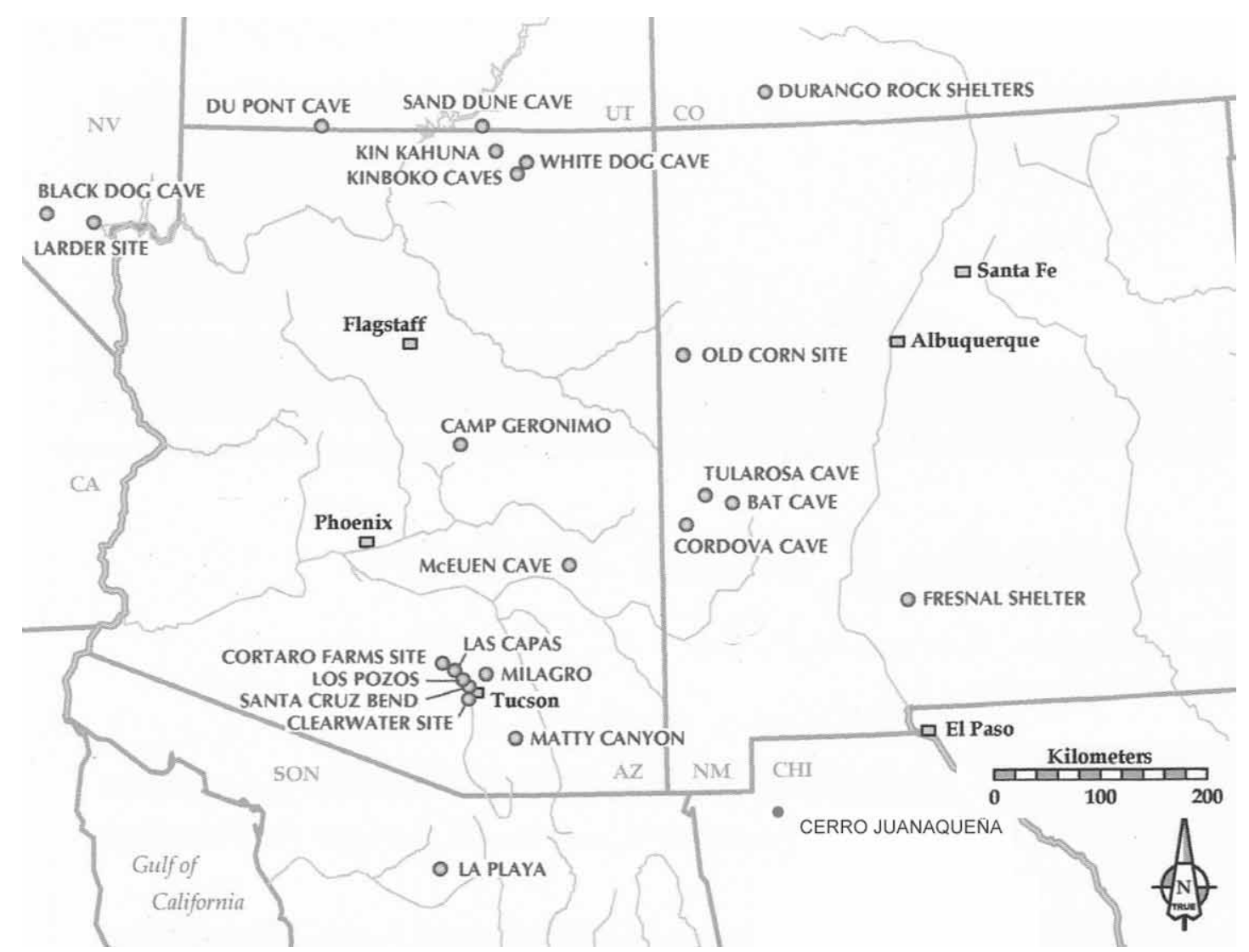

Figura 3. Sitios con maíz más temprano en el suroeste de Estados Unidos y norte de México (tomado de Herr, 2009: 1).

desierto de Sonora existió un abandono, ya que hasta la fecha no existe ninguna fecha de radiocarbono cultural para este período. También sugerimos que el abandono del desierto dio como resultado la bifurcación de la población protoyutoazteca en las ramas yutoaztecas norteña y sureña: algunos grupos buscaron refugio en la Gran Cuenca, y otros se movieron hacia el sur, tal vez a la región serrana entre los ríos Mayo y Sinaloa. Miller (1983) ha identificado esta zona como la cuna de la rama sureña de los grupos yutoaztecas. Proponemos que esos grupos protoyutoaztecas fueron los primeros "norteños" que adoptaron el maíz durante los últimos años/siglos del período Altitermal, y tal vez por las mismas razones propuestas por los modelos difusionistas: cambios ambientales y/o aumento de la población que resultó de la concentración de la población en áreas restringidas. Es muy probable que la raza Chapalote fuera desarrollada entre los protoyutoaztecas sureños.

\section{El período de Agricultura Temprana y el sitio de La Playa}

Hasta la fecha el sitio de La Playa (SON F:10:3), ubicado 10 kilómetros al norte de estación Trincheras y unos $37 \mathrm{~km}$ al suroeste de Santa Ana (Figura 3), sigue siendo el único sitio de agricultura temprana estudiado en el estado de Sonora (Carpenter, 2009; Carpenter et al., 2009, 2007, 2005, 2003 y 2002). Los datos obtenidos indican que el valle del río Boquillas fue utilizado esporádicamente desde época Paleoindia pero durante el Holoceno tardío, y que la ocupación del valle de Boquillas se incrementó considerablemente en el Holoceno tardío (2500 a.C.-200 d.C.), lo cual parece coincidir con el regreso de condiciones climáticas más favorables. La población del valle se incrementó notablemente en el período Agricultura Temprana, que comenzó alrededor de 1500 a.C. con dos fases: la San Pedro (ca. 1500/1200 a 800 a.C.) y la Ciénega (800 a.C.-ca. 


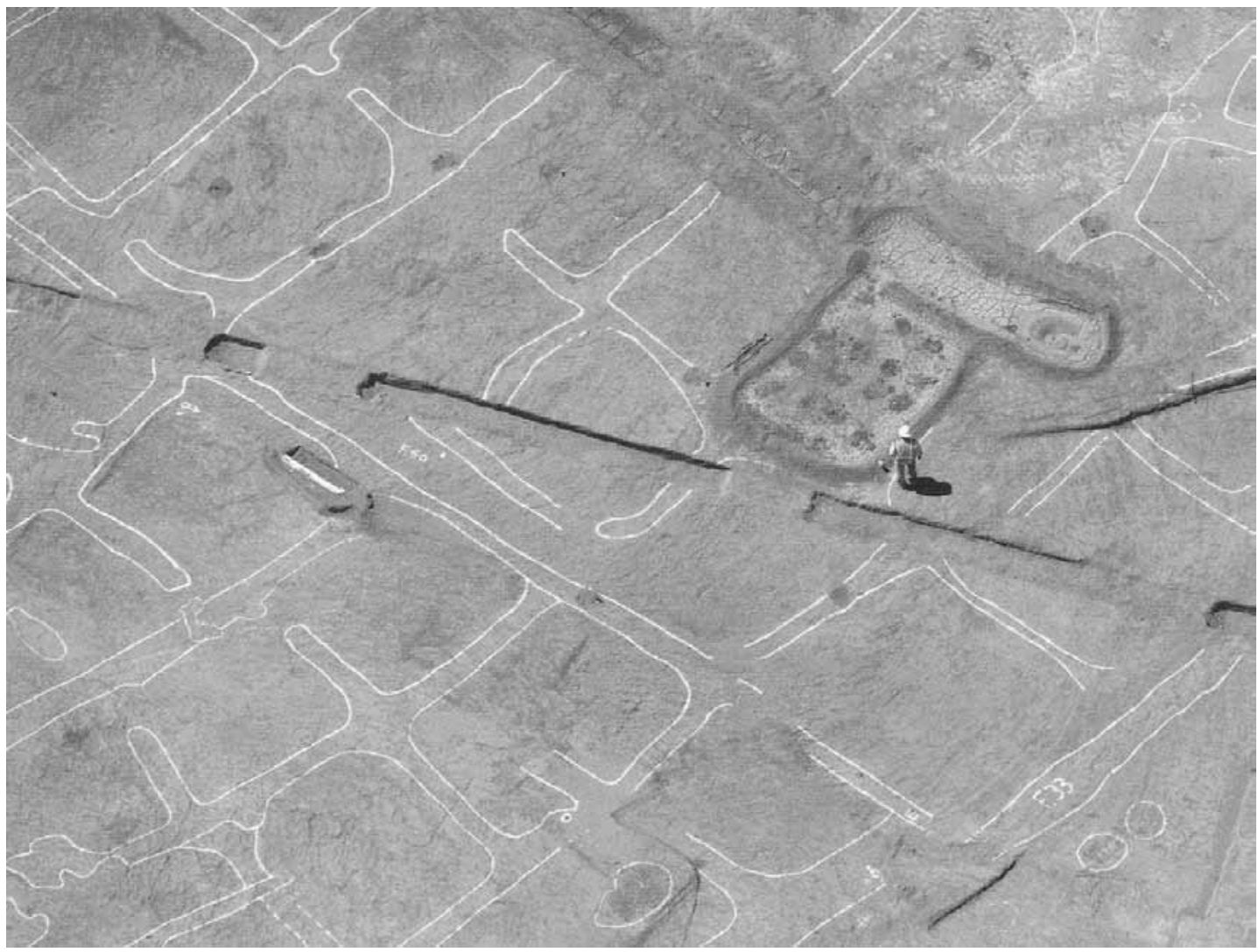

Figura 4. Campos de cultivo con bordos en el sitio de Las Capas (Herr, 2009:1).

200 d.C), caracterizadas por puntas de tipos Imperio, San Pedro y Ciénega. La fecha más temprana que tenemos registrada para este período es de $3720+/-320$ (elemento 323) de un entierro femenino adulto flexionado, la única fecha directa de maíz es de un grano que se encontraba dentro de un hoyo de poste con una edad de radiocarbono de 1885+/-50. Estudios recientes geomorfológicos y geocronológicos sugieren que una vez que terminó el período Altitermal (4500 años calibrados a.P.), el sitio de La Playa fue ocupado por grupos humanos sin interrupción; por lo menos en dos perfiles realizados en el sitio se encontraron superficies de ocupación con restos de carbón que fueron fechadas a 4330+/-90 y 4160+/-80 (Copeland et al., 2012:2937). Esta evidencia parece indicar que se trata de yutoaztecanos probablemente con maíz que llegaron a ocupar el valle del río Boquillas. Recientemente llevamos a cabo un proyecto de investigación específicamente para encontrar los maíces más tempranos de La Playa estudiando los contextos post-Altitermales más tempranos, una vez que la estratigrafía del sitio ya ha sido establecida por un grupo de geocientíficos de la Universidad de Arizona (Copeland et al., 2012)

\section{Los yutoaztecas sureños, la sequía del Altitermal y la bonanza post-Altitermal}

Por algún tiempo hemos propuesto que la difusión de maíz en el noroeste de México y el suroeste de los EE.UU. puede ser atribuida a la dispersión de grupos hablantes de yutoaztecas que ocurrió durante los primeros siglos inmediatamente después del período Altitermal (Holoceno medio), aunque algunos investigadores siguen debatiendo la severidad de las condiciones ambientales; parece ser que por lo menos en el desierto de Sonora las condiciones adversas provocaron un abandono poblacional sustancial. También proponemos que el abandono del desierto dio como resultado la bifurcación de la población protoyutoazteca en 
las ramas yutoaztecas norteña y sureña: algunos grupos buscaron refugio en la Gran Cuenca, y otros se movieron hacia el sur, tal vez a la región serrana entre los ríos Mayo y Sinaloa. Miller (1983) ha identificado esta zona como la cuna de la rama sureña de los grupos yutoaztecas. Proponemos que grupos protoyutoaztecanos sureños empezaron a formar un continuo cultural desde aproximadamente 6000 a.C. y fueron los primeros "norteños" que adoptaron el maíz durante los últimos años/siglos del período Altitermal, y tal vez por las mismas razones propuestas por los modelos difusionistas: cambios ambientales y/o aumento de la población que resultó de la concentración de la población en áreas restringidas que fue seguido por un período pluvial con un incremento de la precipitación y los mantos de agua.

El inicio del Holoceno tardío, alrededor del 2500 a.C., marcó un período pluvial de condiciones ambientales más favorables. Posiblemente, el desierto de Sonora que permanecía como un nicho vacío, pudo ser reocupado por estos grupos de agricultores protoyutoaztecas que dejaron sus refugios. Estos movimientos poblacionales pudieron haber contribuido a la diversificación del yutoaztecano; por medio de la glotocronología, los lingüistas han propuesto que la variación comenzó por esta época. Cabe mencionar que la distribución de sitios de agricultura temprana del desierto de Sonora concuerda con el área ocupada por grupos yutoaztecas de la rama sureña al momento del contacto en el siglo XVI.

\section{Proposiciones sobre la época post-Altitermal y el desarrollo del Período de Agricultura temprana visto desde los llanos de Hermosillo y zonas aledañas}

Recientemente hemos podido documentar que en varios sitios arqueológicos arcaicos localizados en la subprovincia del desierto de Sonora de los llanos de Hermosillo, la existencia de depósitos estratigráficos del período Altitermal y/o la época fluvial pos-Altitermal (Sánchez, 2010). En los sitios de El Bajío, El Aigame, El Aguajito, el Fin del Mundo, y La Playa existen registros de black mats o depósitos de ciénagas o humedad que fechan entre 5000-4000 años de radiocarbono antes del presente junto con evidencias de ocupación humana (Sánchez, 2010) (Figura 5). La formación de estas ciénagas es un testimonio de que hacia el final del período Altitermal existió un período de humedad relativamente alta que recargó los mantos acuíferos y favoreció la formación de ciénagas. Estas condiciones ambientales favorables permitieron que los grupos humanos del Arcaico volvieran a ocupar las zonas bajas de Sonora y comenzaron a experimentar con el cultígeno mesoamericano maíz con el que se familiarizaron cuando tuvieron contacto con los grupos yutoaztecanos sureños.

\section{El sitio de El Bajío}

Desde 1997 en el sitio de El Bajío se observó un depósito de black-mat que se pensó pertenecía a la época del Pleistoceno terminal/Holoceno tardío en la localidad 11; al fechar el depósito resultó ser una ciénaga con una fecha de 5390+/-120 años antes del presente. Dos puntas San José fueron recolectadas de la superficie (Sánchez Morales, 2012) y no hay evidencias contundentes sobre el establecimiento de grupos humanos durante el Holoceno temprano. Hasta el momento se cuenta con cuatro fechamientos por radiocarbono que caen dentro del Arcaico medio en un rango entre $5390+/-120$ y $3560+/-40$ años a.P., siendo esta última fecha de especial interés, pues corresponde a una lentícula de ceniza hallada dentro de un estrato de arena y grava en la Cala 8 de la Localidad 6, directamente asociada a desechos de talla (Sánchez, 2010: 157). Esta información contribuye firmemente al planteamiento de una ocupación durante el Arcaico medio en El Bajío.

\section{El sitio del Aígame}

Se localiza a 59 kilómetros al este de Hermosillo en la cuenca del río Mátape. El Proyecto Geoarqueología y Tecnología Lítica de los sitios paleoindios de Sonora registró cuatro diferentes depósitos de ciénagas del final del Altitermal. Tres puntas de proyectil pertenecientes a este período del tipo San José están presentes en dos localidades del sitio (Sánchez Morales, 2012). En la localidad 3 se encontró un grueso depósito de ciénaga, de la parte baja del depósito se obtuvo una fecha de carbón de 4009 +/-42.

Se tomó un perfil de polen de este pozo que fue analizado por la arqueobotánica Susana Xelhuantzi de la Subdirección de Laboratorios y Apoyo Académico. La concentración de polen fue muy baja, porque las muestras tenían un alto contenido de sílice. Sin embargo, las muestras 


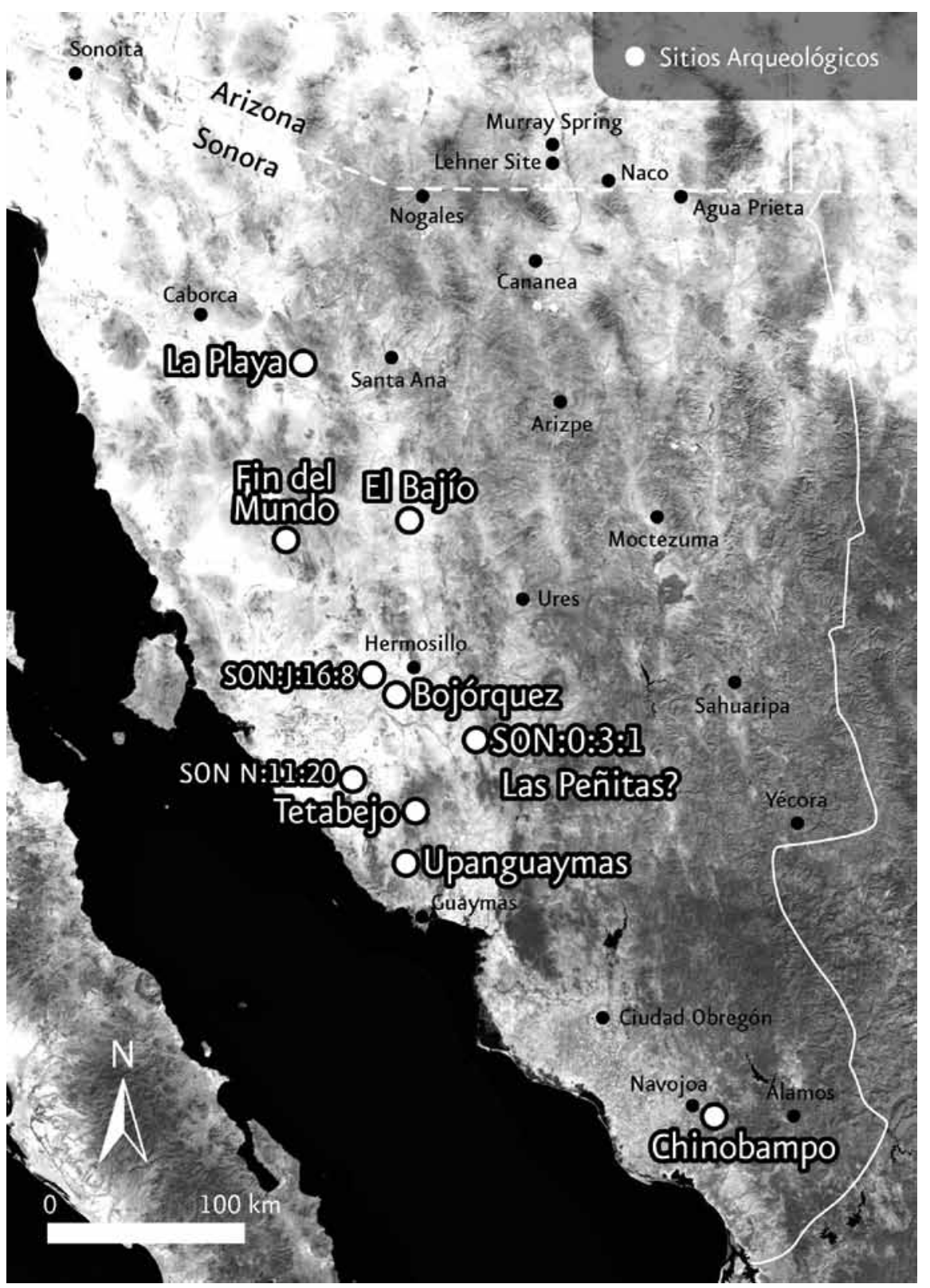

Figura 5. Sitios en Sonora con depósitos post-Altitermales.

revelaron que se trata de un depósito de ciénaga que contiene algas y plantas acuáticas consistentes con un ambiente húmedo (Xelhuantzi, 2008). En la muestra obtenida de la base de la ciénaga Xelhuantzi encontró un fitolito de maíz (Figura 6). Aunque se necesitan hacer más estudios y tomar muchas más muestras parece que El Aígame es un sitio de importancia para entender el principio de las comunidades agrícolas en Sonora.

\section{El sitio del Aguajito}

El sitio se encuentra a 40 kilómetros al este de Hermosillo, también en la cuenca del río Mátape. Este es un sitio similar a La Playa con erosiones en cárcavas y con un gran número de hornos y piedras quebradas por fuego en la superficie. En varios perfiles del sitio se observaron depósitos de ciénagas, estos depósitos fueron fechados a 


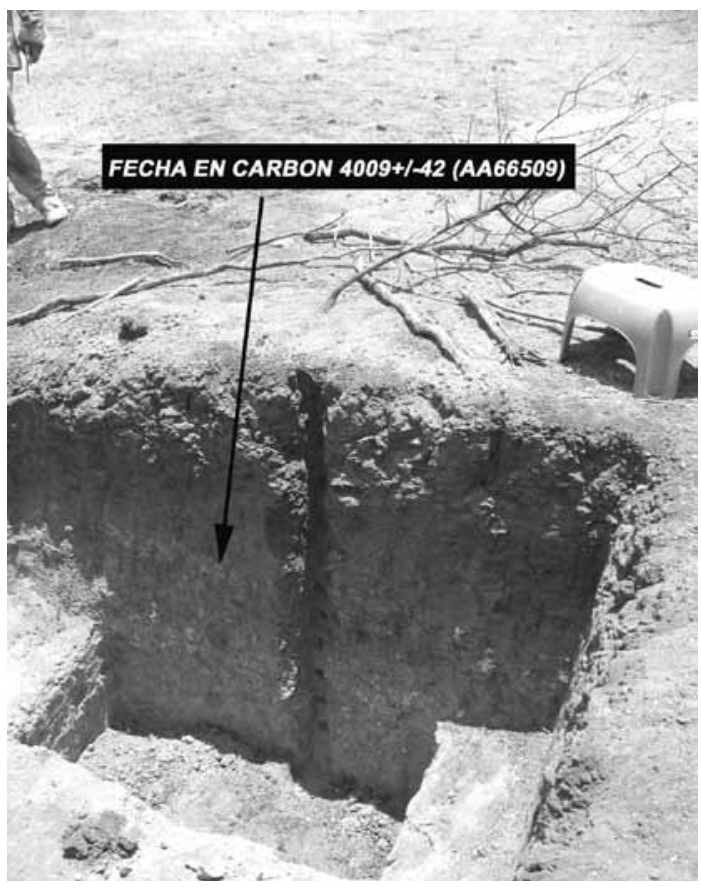

Figura 6. Perfil de polen con fecha de 4000 años donde se encontró un fitolito de maíz.

4507+/-45 con una fecha de carbón, también se tomaron tres muestras de suelo concordantes con la fecha de carbón (Figura 7). Este sitio parece tener depósitos estratificados de los últimos 5000 años y punta de proyectil pertenecientes al arcaico medio y tardío han sido recolectadas del sitio. Aquí se pretende realizar un recorrido sistemático del sitio y excavar hornos que nos den información sobre los contextos primarios y tomar muestras para fechamiento, restos paleobotánicos y polen en el caso que sea posible.

\section{El Fin del Mundo}

El Fin del Mundo se localiza en la cuenca del río Bacoachi a 120 kilómetros al norte de Hermosillo. La ocupación del Arcaico medio en el Fin del Mundo está bien representada con por lo menos 50 puntas Pinto/San José (Figura 7). Una fecha obtenida de una cuenta hecha de concha nacarada encontrada en un contexto alterado dio una fecha de 4,840+-40 (AA83955). El sitio tiene una gran cantidad de hornos, por lo que se pretende excavar algunos hornos en dos localidades arcaicas que han sido registradas para tomar muestras arqueobotánicas y, en el caso en el que sea posible, muestras de polen para buscar maíz.

\section{Conclusiones}

Las investigaciones de diferente índole realizadas hasta la fecha en Sonora han dejado de manifiesto que por lo menos media docena de sitios diferentes contienen depósitos estratificados de los últimos 5000 años. Todos estos sitios además de contener depósitos de los últimos 5000 años donde se observa esta época de humedad también presentan evidencias de elementos arqueológicos de esta época. En el

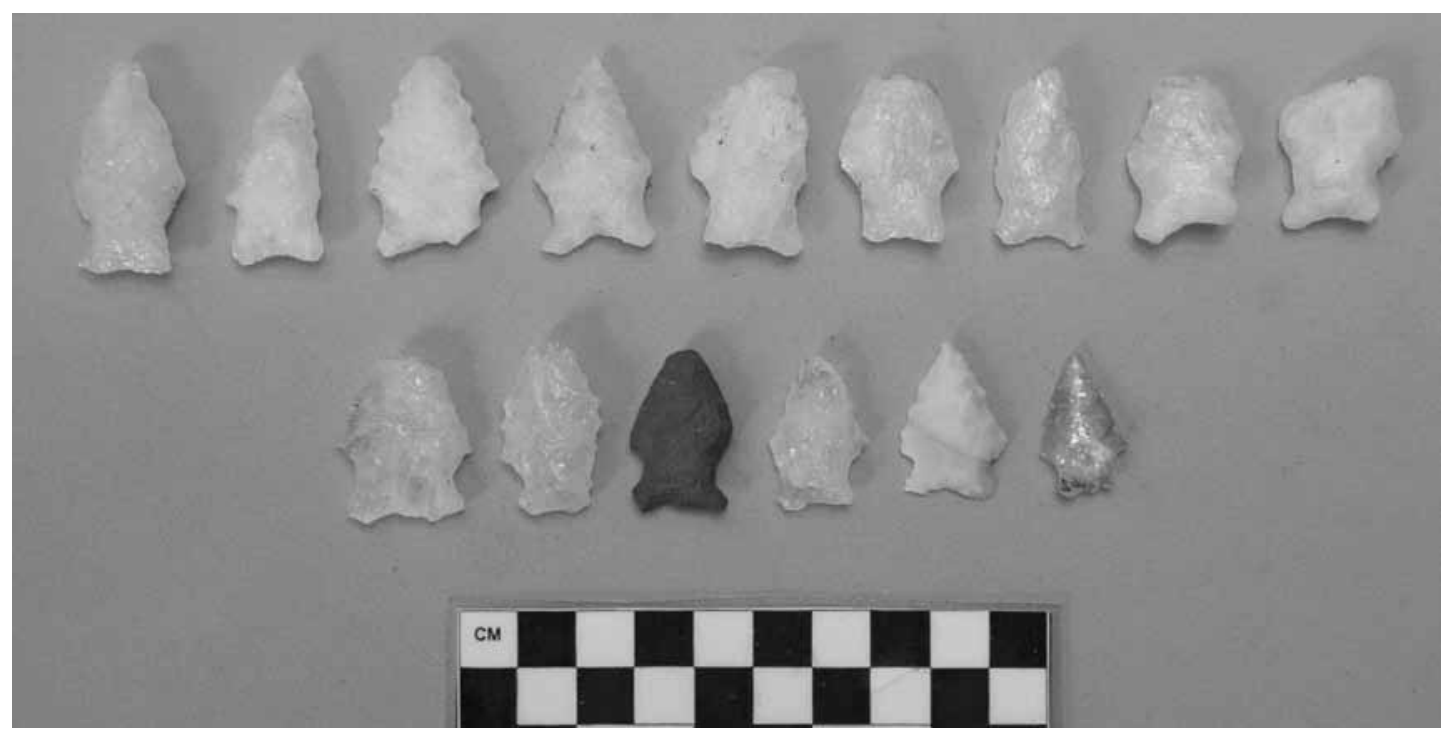

Figura 7. Puntas Pinto y San José del sitio Fin del Mundo. 
sitio de El Aígame se encontró un fitolito de maíz en una muestra tomada del pozo 5 (Xelhuatzin, 2008) en los depósitos de una ciénaga con fecha de 4000 años. El estudio integral de los contextos post-Altitermal en estos sitios en y alrededor de los llanos de Hermosillo será una contribución fundamental para el estudio del período cuando el maíz llegó a Sonora, los movimientos de los grupos yutoaztecas después del período Altitermal y el estudio de la transición de los grupos humanos de una vida cazadora-recolectora a una vida agricultora. Seguramente la planicie de Sonora funcionó como un corredor cultural que permitió la transmisión del maíz al suroeste de Estados Unidos.

\section{Referencias Citadas}

Antevs, Ernst

1948 The Great Basin, with Emphasis on Glacial and Postglacial Times. University of Utah Bulletin 38(20): 168-191.

1955 Geologic Climatic Dating in the West. American Antiquity 20: 317-355.

Benz, B.F.

1994 Reconstructing the Racial Phylogeny of Mexican Maize: Where do We Stand?. En Corn and Culture in the Prehistoric New World, editado por S. Johannessen y C.A. Hastorf, pp. 23-33. Westview Press, Boulder.

1999 On the Origin, Evolution, and Dispersal of Maize. En Pacific Latin America in Prehistory: The Evolution of Archaic and Formative Cultures, editado por M. M. Blake, pp. 25-38. Washington State University Press, Pullman.

2001 Archaeological Evidence of teosinte Domestication from Guilá Naquitz, Oaxaca. Proceedings of the National Academy of Sciencie, 98(4): 2104-2106.

Berry, C.F. and M. S. Berry

1986 Chronological and Conceptual Models of the Southwestern Archaic. En Anthropology of the Desert West: Essays in Honor of Jesse D. Jennings, editado por C.J. Condie and D.D. Fowler, pp. 253-327. Anthropological Papers No. 110. University of Utah Press, Salt Lake City.

Betancourt, Julio

1990 "Late Quaternary Biogeography of the Colorado Plateau", en Packrat Middens: The Last 40,000 Years of Biotic Change, editado por J.L. Betancourt, T.R. VanDevender y P.S. Martin, Tucson, University of Arizona Press, pp. 259-292.

Carpenter, John; Guadalupe Sánchez y Elisa Villalpando 1996 Of Language, Lithics and Lunch: New Perspectives on the San Pedro Phase from La Playa, Sonora, Mexico, Ponencia presentada en Conference on Archaic Prehistory of the Southwest, Albuquerque.

1998 De los Tiempos y el Río: Investigaciones Preliminares en el sitio de La Playa, Sonora. En Antropología e Historia del Occidente, Memorias de la XXIV Mesa Redonda de la Sociedad Mexicana de Antropología, pp. 985-1010. SMA, México, D. F.

2002 Of Maize and Migration: Mode and Tempo in the Diffusion of Zea mays in Northwest Mexico and the American Southwest. En Traditions, Transitions, and Technologies: Themes in Southwestern Archaeology, editado por Sarah Schlanger, pp. 245-258. University of Colorado Press, Boulder.

2005 The Late Archaic/Early Agricultural Period in Sonora, Mexico. En New Perspectives on the Late Archaic Across the Borderlands, editado por Bradley J. Vierra, pp. 3-40. University of Texas Press, Austin.

2007 El Período de Agricultura Temprana en Sonora. Memoria del Seminario de Arqueología del Norte de México, editado por Cristina García M. y Elisa Villalpando C. pp. 59-78.
Coordinación Nacional de Arqueología/Centro INAH Sonora. Hermosillo, Sonora.

Carpenter, John; Jonathan Mabry y Guadalupe Sánchez 2001 Arqueología de los Grupos Yutoaztecas Tempranos. En Avances y Balances de las Lenguas Yutoaztecas: Homenaje a Wick R. Miller, editado por José Luis Moctezuma y Jane H. Hill, pp. 359-374. Colección Científica 438. INAHCONACULTA, México, D.F.

Copland, Audrey; Jay Quade; James T. Watson; Brett T. McLaurin y Elisa Villalpando

2012 Stratigraphy and geochronology of La Playa archaeological site, Sonora, Mexico. Journal of Archaeological Science 39 (2012): 2934-2944

Davis, Owen K.

1984 Multiple Thermal Maxima During the Holocene. Science 225: 617-619.

Doebley, J.F.

1990 Molecular Evidence and the Evolution of Maize. Economic Botany 44: 6-27.

Doebley, J.F.; M.M. Goodman and C.W. Stuber

1984 Isoenzymatic Variation in Zea (Gramineae). Systematic Botany 9: 203-218.

1987 Patterns of Isozyme Variation between Maize and Mexican Annual Teosintle. Economic Botany 41: 234-246.

Fish, S.; P. Fish and J. Madsen

1990 Sedentism and Settlement Mobility in the Tucson Basin prior to A.D. 1000. En Perspectives on Southwestern Prehistory, editado por Paul E. Minnis and Charles L. Redman, pp. 76-91. Westview Press, Boulder.

Fowler, Catherine S.

1983 Some Lexical Clues to Uto-Aztecan Prehistory", en International Journal of Linguistics, vol. 49, pp. 224-257.

Goss, J.A.

1977 Linguistic Tools for the Great Basin Prehistorian. En Models in Great Basin Prehistory, edited by D. D. Fowler, pp. 49-70. Desert Research Institute Publications in the Social Sciences No. 12. Reno.

Hard, R.J.

1986 Ecological Relationships Affecting the Rise of Farming Economies: A Test from the American Southwest. Disertación doctoral no publicada. University of New Mexico, Albuquerque.

Herr, Sarah A.

2009 The Latest Research on the Earliest Farmers. Archaeology Southwest 23(1): 1-3, Center for Desert Archaeology, Tucson, Arizona.

Hill, Jane $\mathrm{H}$.

1996 The Prehistoric Differentiation of Uto-Aztecan Languages and the Lexicon of Early Southwestern Agriculture", ponencia presentada en la 61 reunión anual de Society for American Archaeology, New Orleans, abril. 
2001 Proto-Uto-Aztecan: A Community of Cultivars in Central Mexico? American Anthropologist 103(4): 913-934. Holliday, V.T.

1989 Middle Holocene Drought on the Southern High Plains. Quaternary Research 31: 74-82. 1989b.

Huckell, Bruce

1990 Late Preceramic Farmer-Foragers in Southeastern Arizona: A Cultural and Ecological Consideration of the Spread of Agriculture into the Southwestern United States. Disertación doctoral no publicada. University of Arizona, Tucson.

1995 Of Marshes and Maize: Preceramic Agricultural Settlements in the Cienega Valley, Southeastern Arizona, Anthropological Papers of the University of Arizona, 59.Tucson, University of Arizona Press.

Iltis, H.H.

1983 From Teosinte to Maize: The Catastrophic Sexual Transmutation. Science 222: 186-188.

1987 Maize Evolution and Agricultural Origins. En Grass Systematics and Evolution, editado por T. Soderstrom, K. Hilu, C. Campbell, and M. Barksworth, pp. 195-213. Smithsonian Institution Press, Washington.

Mabry, Jonathan; John Carpenter y Guadalupe Sánchez

2008 Archaeological Models Of Early Uto-Aztecan Prehistory in the Arizona-Sonora Borderlands. En Archaeology Without Borders: Contact, Commerce, and Change in the U.S. Southwest and Northwestern Mexico, editado por Laurie Webster y Maxine McBrinn, pp. 155-184.

Martin, Paul S.

1963 The Last 10,000 Years, Tucson, University of Arizona Press.

McClung De Tapia, E.

1997 La Domesticación del Maíz. Arqueología Mexicana 5(25): 34-39.

Matson, R.G.

1991 The Origins of Southwestern Agriculture. University of Arizona Press, Tucson.

1999 The Spread of Maize to the Colorado Plateau. Archaeology Southwest 13(1): 10-11.

Meltzer, David J.

1991 Altithermal Archaeology and Paleoecology at Mustang Springs, on the Southern High Plains of Texas, American Antiquity 56 (2), pp. 236-267.

Merrill, William L.; Robert J. Hard; Jonathan B. Mabry; Gayle J. Fritz; Karen R. Adams; John R. Roney and A.C. Macwilliams 2009 The diffusion of maize to the southwestern United States and its impact. PANS,vol.106_no.50_21019-21026 Miller, W.R.

1983 Uto-Aztecan Languages. En Handbook of North American Indians, Vol. 10, editado por A. Ortiz, pp. 113124. Smithsonian Institution, Washington, D.C.

1995 Prehistoria de las lenguas indígenas del noroeste de México. En Sonora: Origen y Destino, Memoria de XIX Simposio de Historia y Antropología de Sonora, Vol. 2, pp. 167-171. University of Sonora, Hermosillo.

Nabhan, Gary P.

1985 Gathering the Desert. University of Arizona Press, Tucson.

1989 Enduring Seeds: Native American Agriculture and Wild Plant Conservation. North Point Press, San Francisco.

Noguera, Eduardo

1958 Reconocimiento arqueológico en Sonora, México, Dirección de Monumentos Prehispánicos INAH (Informe, 10).
Niederberger, Cristine

1976 Zohapilco: Cinco Milenios de Ocupación Humana en un Sitio Lacustre de la Cuenca de México, México. Instituto Nacional de Antropología e Historia; Ciudad de México, México.

1979 Early Sedentary Economy in the Basin of Mexico. Science 203: 131-142.

Nichols, M.J.P.

1981 Old California Uto-Aztecan. Survey of California and Other Indian Languages, Reports 1: 5-41.

Piperno, D.R. y K.V. Flannery

2001 The Earliest Archaeological Maize (Zea mays L.) from Highland Mexico: New Accelerator Mass Spectrometry Dates and their Implications. Proceedings of the National Academy of Science 98(4): 2101-2103.

Romney, A.K.

1957 The Genetic Model and Uto-Aztecan Time Perspective. Davidson Journal of Anthropology 3: 35-41.

Roney, John R. y Robert J. Hard

1999 Northwestern Mexico: New Perspectives on the Late Archaic from Cerro Juanaqueña. Ponencia presentada en 64ava Reunión de la Society for American Archaeology, Chicago, E.U.A.

Sánchez, Guadalupe

2010 Los Primeros Mexicanos: Late Pleistocene/Early Holocene Archaeology in Sonora. Tesis de Doctorado no publicada del School of Anthropology de la University of Arizona.

Sánchez González, J.

1994 Modern Variability and Patterns of Maize Movement in Mesoamerica. En Corn and Culture in the Prehistoric New World, editado por S. Johannessen and C. A. Hastorf, pp. 135-156. Westview Press, Boulder.

Sánchez Morales, Ismael

2012 Estudio de las Puntas de Proyectil y Bifaciales de los Sitios de Sonora. Tesis de Licenciatura no publicada. Escuela Nacional de Antropología e Historia, D.F., México.

Turner, R.M. y D.E. Brown

1994 Tropical-Subtropical Desertlands: Sonoran Desertscrub. En Biotic Communities Southwestern United States and Northwestern Mexico, editado por D. E. Brown, pp.180222. University of Utah Press, Salt Lake City, Utah.

Van Devender, T.R. y W.C. Spaulding

1979 Development of vegetation and climate in the southwestern United States. Science 204: 701-710.

Van Devender, T.R.; Thompson, R.S. y Betancourt, J.L.

1987 Vegetational history in the Southwest: The nature and timing of the late Wisconsin-Holocene transition. En North America and Adjacent Oceans During the Last Deglaciation, editado por Ruddiman, W.F. y H.E. Wright Jr, pp. 323-352. Geology of North America, Volume K-3, Geological Society of America, Boulder.

Wills, W.H.

1988 Early Prehistoric Agriculture in the American Southwest. School of American Research Press, Santa Fe.

1999 Bat Cave. Archaeology Southwest 13(1): 13.

Xelhuantzi López, María Susana

2008 Informe del Análisis de Polen de dos Perfiles Estratigráficos en el Sitio Clovis de El Aígame, Sonora. Reporte archivado en Subdirección de Laboratorios y Apoyo Académico, Coordinación Nacional de Arqueología, INAH, México. 